\title{
Ultasonographic indicators of carpal tunnel syndrome demonstrate reversibility following carpal tunnel release
}

\author{
Ryusuke Osada*, Mineyuki Zukawa and Tomoatsu Kimura \\ Department of Orthopedic Surgery, Faculty of Medicine, University of Toyama, Japan
}

\begin{abstract}
Background: The objective of the present study was to use ultrasound examination to determine the cross-sectional area (CSA) of the median nerve and quantify transverse sliding motion during finger movements in patients with carpal tunnel syndrome (CTS).

Method: CSA and transverse sliding motion were compared between 15 cases with bilateral CTS (30 hands; 3 men, 12 women; average age: 65.7 years) and a control group (30 hands) of age- and sex-matched healthy individuals. Furthermore, 13 hands of 12 patients who underwent open carpal tunnel release ( 2 men, 10 women; average age: 62.3 years) were assessed and compared before and after surgery.

Results: The mean CSA was larger in the CTS group $\left(16.9 \mathrm{~mm}^{2}\right)$ than in the controls $\left(10.0 \mathrm{~mm}^{2}\right)$, whereas transverse sliding motion was significantly higher in controls $(6.6 \mathrm{~mm})$ than in the CTS group $(4.9 \mathrm{~mm})$. The 12 patients with CTS who underwent surgery showed a mean preoperative CSA of $19.1 \mathrm{~mm}{ }^{2}$ that declined significantly to $14.2 \mathrm{~mm}^{2}$ postoperatively, and the mean transverse sliding motion increased from $3.2 \mathrm{~mm}$ preoperatively to $5.0 \mathrm{~mm}$ postoperatively.

Conclusion: Combining CSA and transverse sliding motion as observed in ultrasound examination can improve CTS diagnosis. Increased CSA and decreased transverse sliding motion noted at the median nerve in the wrists of patients with CTS are partially reversible by open carpal tunnel release.
\end{abstract}

\section{Introduction}

Ultrasonography is a useful tool for depicting the median nerve at the wrist. In a hand with carpal tunnel syndrome (CTS), Ultrasonography can show the enlarged median nerve just proximal to the carpal tunnel [1]. In open carpal tunnel release surgery, the enlarged median nerve is recognized as a pseudoneuroma. The location and shape of the median nerve change with passive and active finger movements, and these changes can be observed in real-time with ultrasonography [2-5]. Toge et al. [6] have further investigated median nerve motion in normal subjects during active flexion of each finger. Nakamichi et al. [7] showed that transverse sliding motion was 1.7 $\mathrm{mm}$ with passive index finger movements in healthy individuals, but only $0.4 \mathrm{~mm}$ in patients with CTS, a small difference of $1.3 \mathrm{~mm}$. Hsieh et al. [8] reported similar findings, though they did not give precise transverse sliding motion distances.

The objectives of the present study were to determine the CSA of the median nerve, to quantify maximal TS-motion during finger movements, and to observe the changes of CSA and transverse sliding motion just proximal to the carpal tunnel. These findings were then compared between normal and CTS hands, both before and after surgery.

\section{Patients and methods}

\section{Subjects}

Patients were excluded from the study if they had a history of wrist injury or a disorder affecting the wrist. The study protocol was approved by the Human Ethics Review Committee of our institute, and written, informed consent was obtained from each subject. The CTS group comprised 30 hands from 15 individuals (three men, 12 women; average age 65.7 years) with a confirmed bilateral sensory disorder, pain, numbness, or prolonged latencies of the median nerve on electrophysiological tests. The control group comprised 30 hands of 15 healthy individuals who were age- and sex-matched with the CTS group (three men, 12 women; average age 64.9 years). Individuals in the control group were patients with complaints that included low back or knee pain.

Thirteen hands of 12 patients (two men, 10 women; average age 62.3 years) with CTS who underwent open carpal tunnel release surgery were examined with ultrasound preoperatively and again six months after surgery.

\section{Measurement methods}

The ultrasonography console was a Hi Vision Avius with a $14-\mathrm{MHz}$ linear probe (Hitachi Medical Corp., Tokyo, Japan). The data collection began with the investigator and participant sitting on the opposite sides of a table facing each other. The participant placed his or her forearm in the supine position flat on the table. Ultrasonography imaging began by placing the probe on the forearm perpendicular to the wrist crease to obtain cross-sectional images (Figure 1). A cross-sectional image of the median nerve at the wrist was first obtained with the participant's hand in a relaxed state. The CSA was calculated from this image by outlining

Correspondence to: Ryusuke Osada, Department of Orthopaedic Surgery, Faculty of Medicine, University of Toyama, Sugitani, Toyama-City, Japan; Tel: +81-76-434-7353; Fax: +81-76-434-5035; E-mail: osa97@med.u-toyama.ac.jp

Key words: ultrasonography, carpal tunnel syndrome, median nerve, open carpal tunnel release

Received: November 16, 2017; Accepted: December 15, 2017; Published: December 18, 2017 
the perimeter just inside the hyperechoic epineurium using the tracing tool available with the ultrasonography apparatus. The participant was then instructed to perform finger counting movements in which the fingers were flexed individually, as well as hand grasping followed by releasing movements (i.e., hand opening, extension of all 5 fingers). The sessions and ultrasonography screening were recorded with video (25 images per second). As shown in figure 2, finger movements induced the displacement of median nerve (transverse sliding motion: Video S1). We selected 2 images in recorded series, in those images median nerve was located most radial side in one image, and ulnar side in another. The center point of median nerve was decided the midpoint of radial end ulnar ends of median nerve in each image. The distance between the center point of the 2 images was measured as transverse sliding motion.

\section{Statistical processing}

Data obtained from the 30 hands in the CTS group were compared to those obtained from the 30 hands in the control group using the Mann-Whitney U test. Pre- and postoperative measurements were compared using the paired $t$-test.

\section{Result}

Comparison between CTS and control (Video S2: CTS, S3: control)

The median nerves were enlarged in CTS hands (Figure 3). The mean CSA was significantly larger in the CTS group $\left(16.9 \pm 5.3 \mathrm{~mm}^{2}\right)$ than in the control group $\left(10.0 \pm 3.2 \mathrm{~mm}^{2} ; \mathrm{n}=30\right.$ from 15 participants; $\mathrm{p}<0.01$; Figure $4 \mathrm{a}$ ). The mean transverse sliding motion during finger movements was significantly smaller in the CTS group $(4.9 \pm 1.5 \mathrm{~mm})$ than in the control group $(6.6 \pm 1.9 \mathrm{~mm}, \mathrm{p}<0.01$, Figure $4 \mathrm{~b})$.

\section{Comparison between before and after surgery}

The mean preoperative CSA in patients who had CTS and underwent open carpal tunnel release was $19.1 \pm 5.9 \mathrm{~mm}^{2}$, but this declined significantly to $14.2 \pm 3.5 \mathrm{~mm}^{2}$ after surgery $(\mathrm{n}=13$ from 12 patients; $\mathrm{p}<0.01$; Figure $5 \mathrm{a}$ ). The mean transverse sliding motion during finger movements was $5.0 \pm 1.1 \mathrm{~mm}$ after surgery, significantly higher than before surgery ( $3.2 \pm 1.3 \mathrm{~mm} ; \mathrm{p}<0.01$; Figure $5 \mathrm{~b})$.

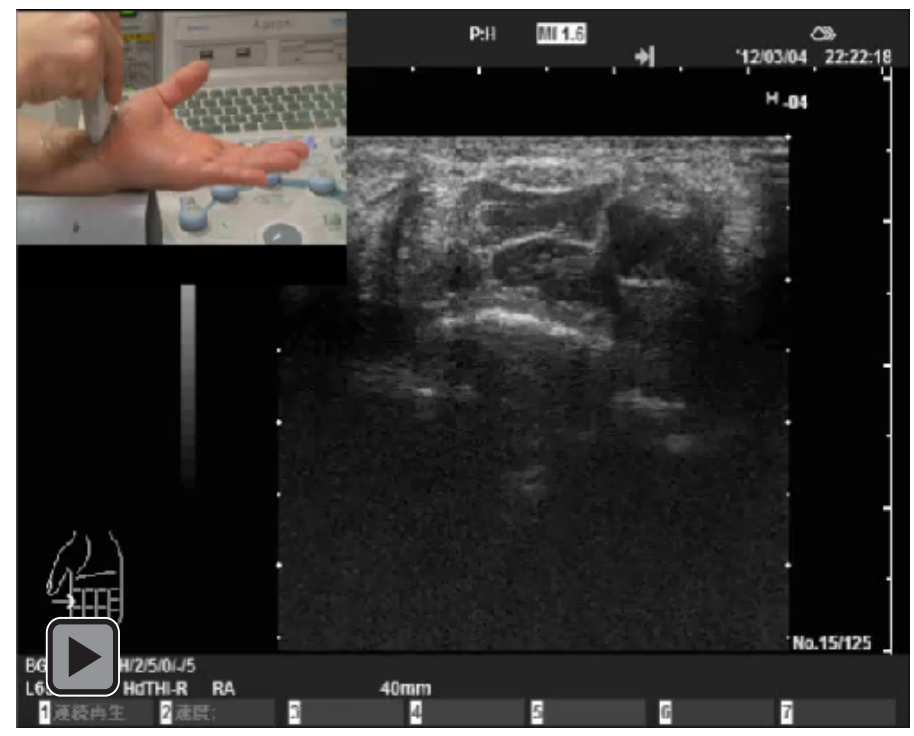

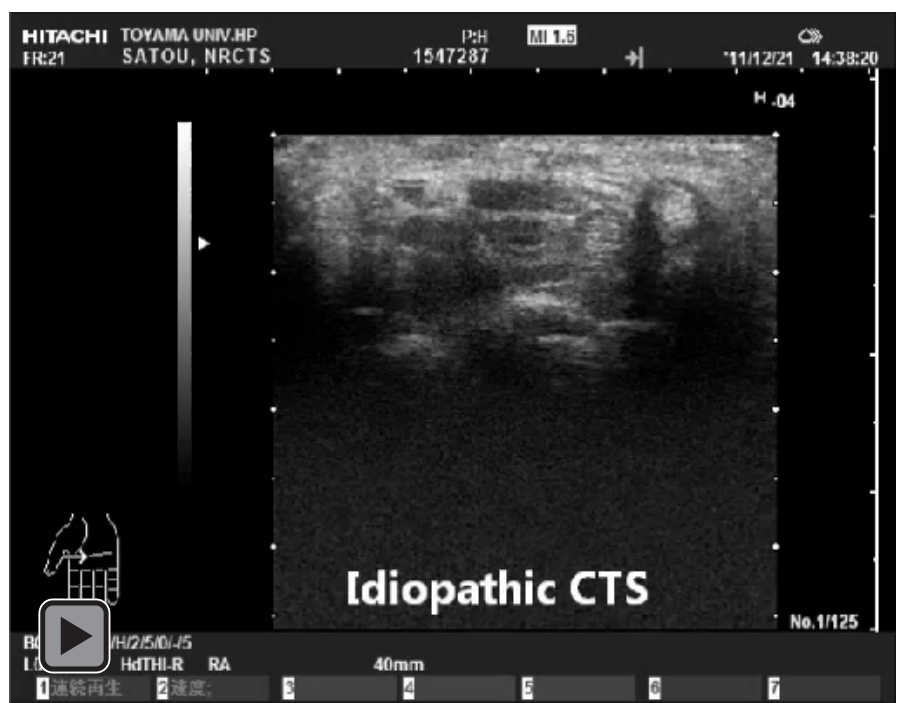

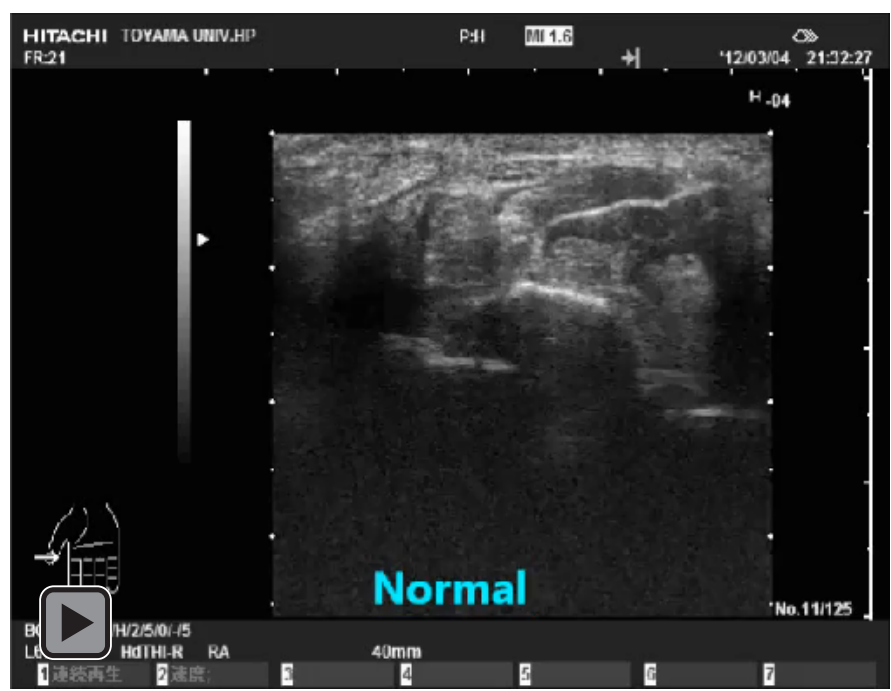

\section{Discussion}

Many reports have described the usefulness of ultrasonography findings of the median nerve for diagnosing CTS [9]. These reports agreed that CTS is characterized by an enlarged CSA of the median nerve at the wrist, and they proposed a diagnostic threshold of 10-12 $\mathrm{mm}^{2}$. The present results are consistent with these previous findings. A major advantage of ultrasonography is its ability to capture nerve movement and shape changes during finger movements in real time. Using ultrasonography images of the carpal tunnel, Nakamichi observed that the median nerve moved in an ulnar-to-radial direction during finger movements, which was called transverse sliding [2]. Yoshii reported that active flexion of only the middle finger produced about $2 \mathrm{~mm}$ of median nerve movement in an ulnar-to-radial direction [3]. Ugbolue reported that, in cadavers, flexing and extending the middle finger produced a maximum movement of 1.4-5.1 mm [10]. In addition, Wang et al. [5] observed median nerve movements with active flexion and extension movements of the fingers and wrist. In that study, 

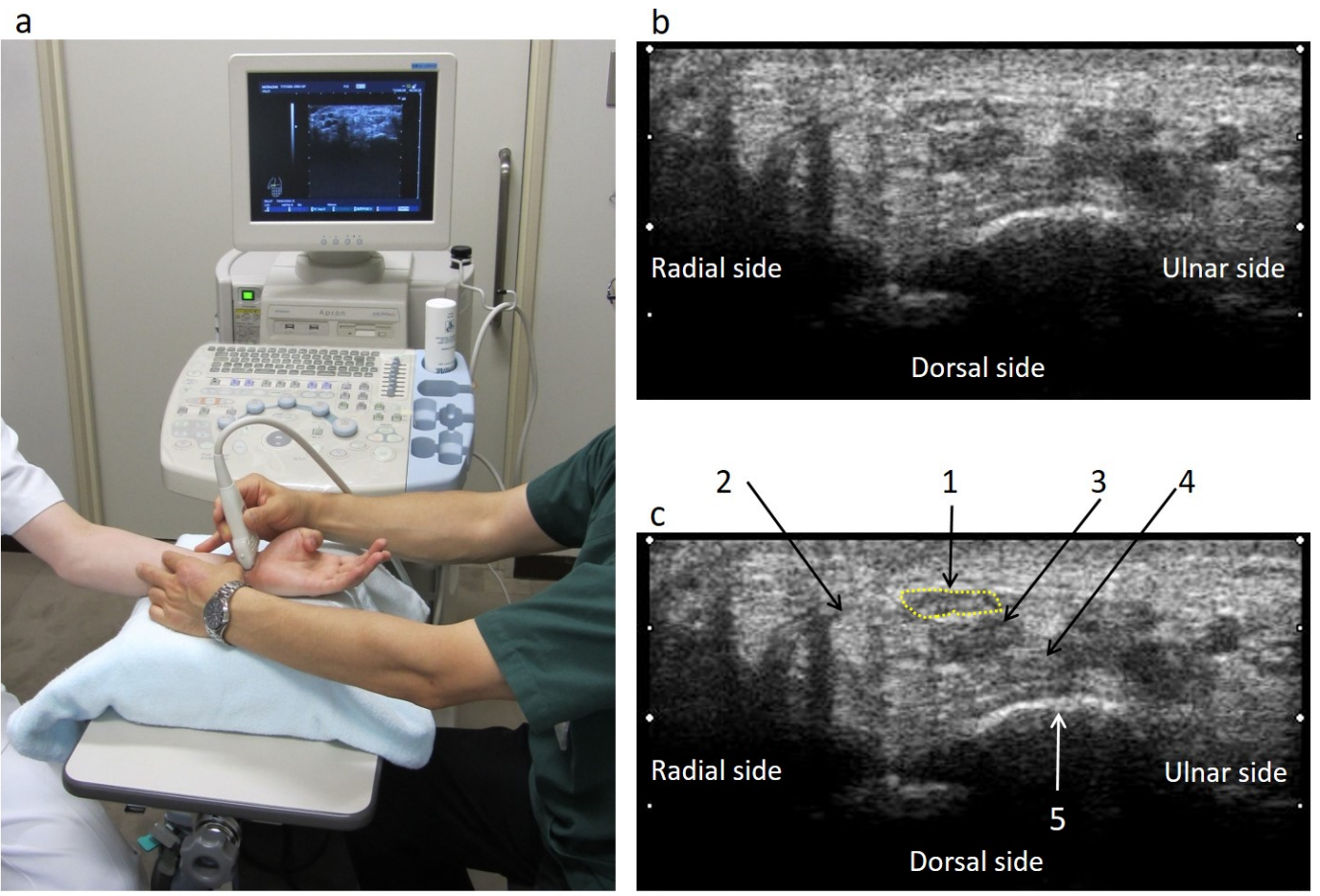

Figure 1. a: Position and posture during ultrasound (US) examination; b: US image obtained in (a) 1: median nerve, 2: flexor pollicis longus tendon, 3: flexor digitorum superficialis tendons, 4: flexor digitorum profundus tendons, 5: lunate.
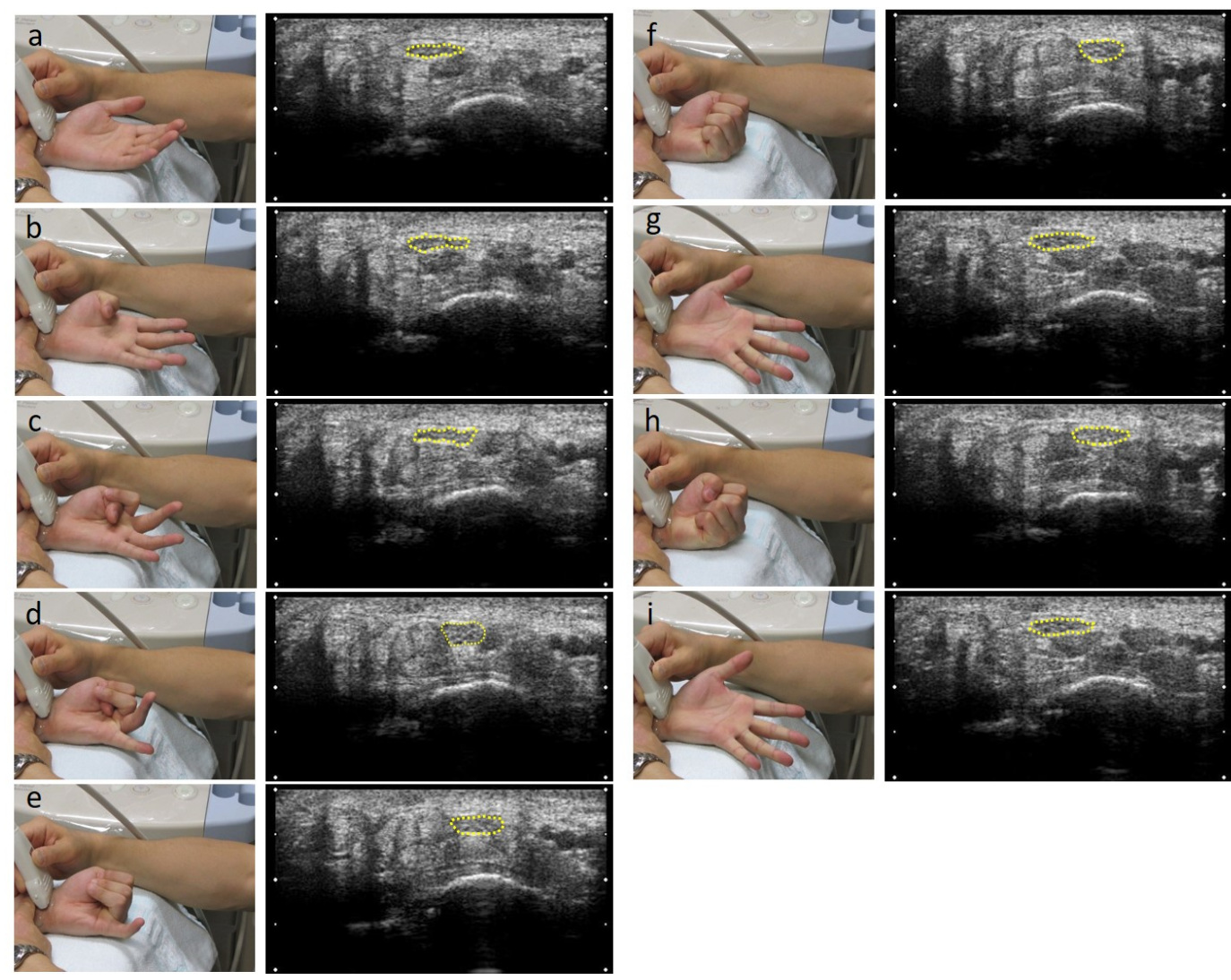

Figure 2. Finger movements and their US images

$\mathbf{a}$ : Hand resting on the table in the supine position (resting position); $\mathbf{b}$ : Thumb flexion (finger count 1); $\mathbf{c}$ : Thumb and index finger flexion (finger count 2); d: Thumb, index finger, and middle finger flexion (finger count 3); e: Thumb, index finger, middle finger, and ring finger flexion (finger count 4); f: Thumb and four fingers flexion (finger count 5); g: Extension of thumb and 4 fingers; h: Gripping movement; i: Extension of thumb and 4 fingers 
a
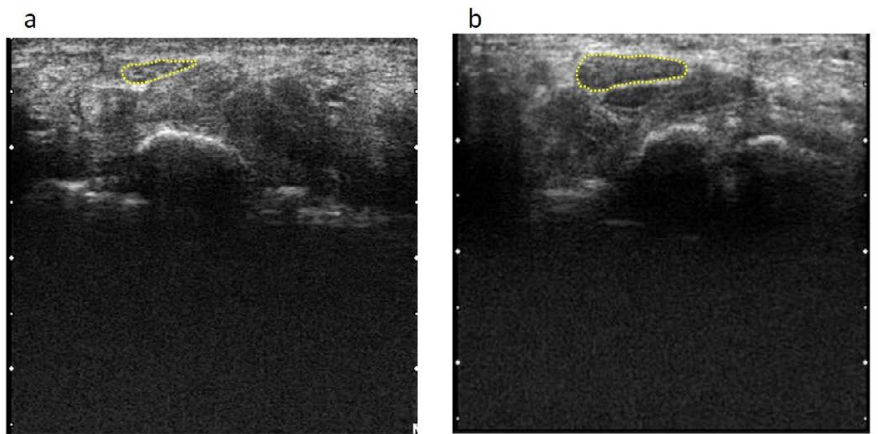

Figure 3. Ultrasonographic images of a healthy individual and a patient with carpal tunnel syndrome (CTS) in the resting position

a: Healthy person's left hand; b: Patient's left hand

The dotted yellow line indicates the border of the median nerve.

a

b
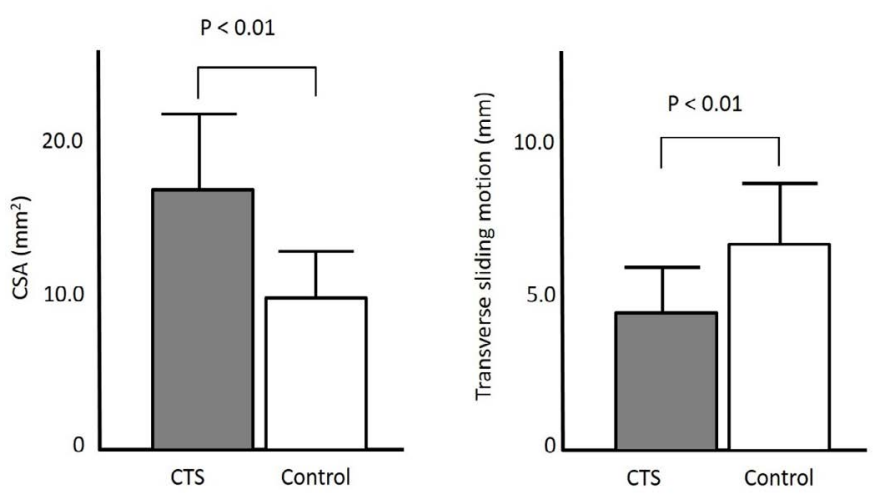

Figure 4. Median nerve CSA (a) and TS-motion (b) in patients with CTS and healthy individuals (mean \pm standard deviation) a

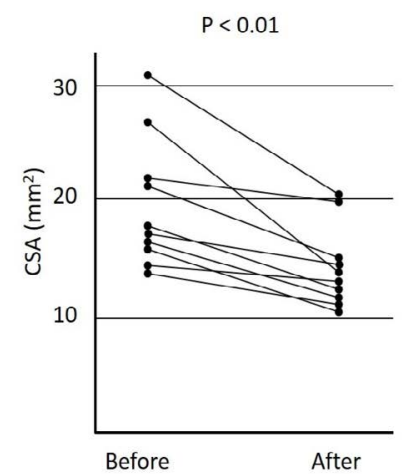

b

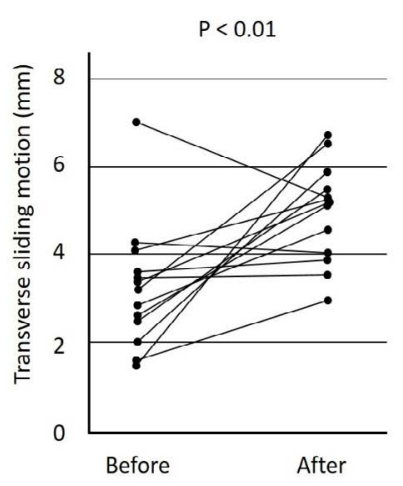

Figure 5. CSA (a) and transverse sliding motion (b) of the median nerve in patients with CTS before and six months after surgery (mean \pm standard deviation)

they reported large nerve movements in the palmar-dorsal direction as well as the ulnar-radial direction. To examine the transverse sliding motion values produced during finger movements in the present study, median nerve movement was measured at the level of the wrist crease (just proximal to the carpal tunnel) in participants performing fingercounting movements in which the median nerve is not compressed by the transverse carpal ligament. The present results showed a mean transverse sliding motion of $6.6 \mathrm{~mm}$ in healthy individuals.
Changes in median nerve mobility have been reported in CTS hands. In healthy individuals, Nakamichi reported transverse sliding motion of $1.75 \mathrm{~mm}$ inside the carpal tunnel during passive index finger movements, but only $0.3 \mathrm{~mm}$ in patients with CTS [7]. Erel used ultrasonography examination to measure median nerve movement at the wrist during passive movements performed with 4 fingers [11]. They observed movement of approximately $1.5 \mathrm{~mm}$ in healthy individuals and a smaller value in patients with CTS. Van Doesburg reported transverse sliding motion of $2 \mathrm{~mm}$ during active middle finger movements [4], whereas Hsieh reported that a gripping action caused the median nerve to enter between the flexor digitorum superficialis and flexor pollicis longus in the hands of healthy individuals, but not in patients with CTS [8]. The present results show that finger movements produced a mean transverse sliding motion of about $6.6 \mathrm{~mm}$ in healthy individuals, which was significantly less in patients with CTS $(4.9 \mathrm{~mm})$. This phenomenon does not conflict with the concept that the mechanisms of CTS involve subsynovial connective tissue inflammation and adhesion.

Compared with healthy individuals, the characteristic ultrasonography findings in patients with CTS were an enlarged CSA and reduced transverse sliding motion. Thus, combining ultrasonography findings regarding the CSA and transverse sliding motion may lead to a more detailed CTS diagnosis.

Ultrasonography is noninvasive, inexpensive, and can be performed quickly and repeatedly. These advantages make ultrasonography a useful tool for making comparisons before and after surgery. Previous reports indicated that the enlarged median nerve CSA seen in CTS can shrink after surgery [12-15], and this is consistent with the present findings. About the displacement of median nerve induced by finger movement, Yoshii reported no significant changes after carpal tunnel release [16]. Our result showed the larger transverse sliding motion of median nerve than before surgery. This disparity might attribute to the differences of measurement methods. In our study, transverse sliding motion after surgery was still smaller than normal.

One limitation of this study is the small number of cases. However, despite the limited number of cases, the postoperative recovery of median nerve transverse sliding motion in patients with CTS was significant. This finding suggests that similar results would be obtained with a larger number of subjects.

The present study shows that enlarged CSA and decreased transverse sliding motion are characteristic of CTS, and these changes are partially reversible. The combined ultrasonography findings regarding the CSA and transverse sliding motion may lead to quicker, less expensive, and more thorough CTS diagnoses, particularly in cases where other diagnostic methods are inconclusive and in cases with unfavorable courses after surgery. Future studies should examine a larger number of cases and standardize the observation conditions so that median nerve pathology can be understood universally.

\section{Conflicts of interest}

All authors hereby declare that they have no conflicts of interest to disclose.

\section{References}

1. Mhoon JT, Juel VC, Hobson-Webb D (2012) Median nerve ultrasound as a screening tool in carpal tunnel syndrome: Correlation of cross-sectional area measures with electrodiagnostic abnormality. Muscle Nerve 46: 871-878. [Crossref]

2. Nakamichi K, Tachibana S (1992) Transverse sliding of the median nerve beneath the flexor retinaculum. J Hand Surg Br 17: 213-216. [Crossref] 
3. Yoshii Y, Villarraga HR, Henderson J, Zhao C, Kai-Nan A, et al. (2009) Ultrasound assessment of the displacement and deformation of the median nerve in the human carpal tunnel with active finger motion. J Bone Joint Surg 91: 2922-2930. [Crossref]

4. van Doesburg MHM, Yoshii Y, Villarraga HR, Henderson J, Cha SS, et al. (2010) Median nerve deformation and displacement in the carpal tunnel during index finger and thumb motion. J Orthop Res 28: 1387-1390. [Crossref]

5. Wang Y, Zhao C, Passe SM, Filius A, Thoreson AR, et al. (2014) Transverse ultrasound assessment of median nerve deformation and displacement in the human carpal tunnel during wrist movements. Ultrasound Med Biol 40: 53-61. [Crossref]

6. Toge Y, Nishimura Y, Basford JR, Nogami T, Yamanaka M, et al. (2013) Comparison of the effects of flexion and extension of the thumb and fingers on the position and crosssectional area of the median nerve. PLoS One 8e: 83565. [Crossref]

7. Nakamichi K, Tachibana S (1995) Restricted motion of the median nerve in carpal tunnel syndrome. J Hand Surg Br 20: 460-464. [Crossref]

8. Hsieh Y-H, Shih JT, Lee H-M, Ho Y-J (2010) Ultrasonography of median nerve mobility in the diagnosis of carpal tunnel syndrome. Formosan Journal of Musculoskeletal Disorders 1: 16-19.

9. Roll SC, Case-Smith J, Evans KD (2011) Diagnostic accuracy of ultrasonography vs. electromyography in carpal tunnel syndrome: a systemic review of literature. Ultrasound Med Biol 37: 1539-1553. [Crossref]
10. Ugbolue UC, Hsu W-H, Goitz RJ, Li Z-M (2005) Tendon and nerve displacement at the wrist during finger movements. Clin Biomech 20: 50-56. [Crossref]

11. Erel E, Dilley A, Greening J, Morris V, Cohen B, et al. (2003) Longitudinal sliding of the median nerve in patients with carpal tunnel syndrome. J Hand Surg Br 28: 439-443. [Crossref]

12. Abicalaf CA, deBarros N, Sernik RA, Pimentel BF, Braga-Baiak A, et al. (2007) Ultrasound evaluation of patients with carpal tunnel syndrome before and after endoscopic release of the transverse carpal ligament. Clin Radiol 62: 891-894. [Crossref]

13. Colak A, Kutlay M, Pekkafali Z, Saraçoglu M, Demircan N, et al. (2007) Use of sonography in carpal tunnel syndrome surgery. A prospective study. Neurol Med Chir (Tokyo) 47: 109-115. [Crossref]

14. Vögelin E, Nüesch E, Jüni P, Reichenback S, Eser P, et al. (2010) Sonographic follow-up of patients with carpal tunnel syndrome undergoing surgical or nonsurgical treatment: prospective cohort study. J Hand Surg Am 35: 140-149. [Crossref]

15. Tas S, Staub F, Dombert T, Marquardt G, Senft C, et al. (2015) Sonographic shortterm follow-up after surgical decompression of the median nerve at the carpal tunnel: a single-center prospective observational study. Neurosurgical Focus 39: E6. [Crossref]

16. Yoshii Y, Ishii T, Tung WL (2015) Ultrasound assessment of the effectiveness of carpal tunnel release on median nerve deformation. J Orthop Res 33: 726-730. [Crossref]

Copyright: @2017 Osada R. This is an open-access article distributed under the terms of the Creative Commons Attribution License, which permits unrestricted use, distribution, and reproduction in any medium, provided the original author and source are credited. 